\title{
Peter Kammerer
}

\section{Die alte DDR - der Mezzogiorno des neuen Deutschland? Ein italienischer Beitrag zur Verfremdung unserer Gegenwart 1}

Zusammenfassung: Es wird am Beispiel der Entwicklungsunterschiede zwichen dem Norden und dem Sïden Italiens nach den Ursachen ihrer Permanenz gefragt, obwohl in Italien seit Jahrzehnten massive staatliche Fördermaßnahmen zu ihrer Einebnung durchgeführt werden. Bei näherer Betrachtung zeigt es sich, daß Unterentwicklung eine Seite von Modernität sein kann, daß also die Entwicklung des Nordens nur die Kehrseite der Zurückgebliebenheit des Südens ist. Mit den Jahr. zehnten verfestigen sich Entwicklungsunterschiede zu einem »einheitlichen Mechanismus*. Der Beitrag versucht, durch „Verfremdung « der deutschen Gegenwart der Frage nach den Chancen der newen Bundesländer nachzugehen, das Niveau der sozioökonomichen Entwicklung der alten Bundesländer in absehbarer Frist zu ereichen. Der Autor kommt zu einer vorläufigen Antwort in Form einer Frage.

Der jüngeren nationalen Geschichte Italiens und Deutschlands ist gemeinsam, daß beide Länder verspätet und darum unter besonderen Schwierigkeiten ihre nationale Einigung volizogen haben und die politische und wirtschaftliche Struktur beider Länder lange vom Neben- und Miteinander einer hochmodernen Industrie mit relativ feudalen Agrarverhältnissen gekennzeichnet war. Das trug zu einer Instabilität demokratischer Strukturen bei und auch dazu, daß sich die allen imperialistischen Ländern gemeinsame Tendenz der Kolonisierung schwächerer Volkswirtschaften mit besonderer Aggressivität nach innen und gegen die unmittelbaren Nachbam richtete. Nach dem zweiten Weltkrieg erlebten beide Länder ihr kapitalistisches Wirtschaftswunder jeweils im halben Land. Heute, nach dem Scheitern des Versuchs einer eigenständigen Entwicklung in der DDR, nehmen die wirtschaftlichen und sozialen Unterschiede zwischen West- und Ostdeutschland Formen an, die erneut an Italien erinnern: nämlich an das italienische NordSüd-Verhältnis mit seinem Einkommensgefälle, seiner unterschiedlichen Ausstattung mit Infrastrukturen und privatem Kapital und mit seinem chronischen Transferbedarf öfentlicher Mittel. Im Jahre 1989 lebten in Süditalien 36,6\% der italienischen Bevőlkerung, 30\% der Beschäftigten und 59,1\% der Arbeitssuchenden. Die Wertschöpfung pro Kopf liegt in Süditalien bei $56 \%$ des norditalienischen Niveaus (SVIMEZ 1990).

1 Es handelt sich um die Kurzfassung eines Referats, das auf einer Konferenz von FU Berlin, Fachbereich Politische Wissenschaft und Humboldt-Universität Berlin, Institut für interdisziplinäre Zivilisationsforschung im Dezember 1990 gehalten wurde. 
Ich möchte im folgenden die Entwicklung des italienischen Nord-Südverhältnisses unter der Fragestellung skizzieren, ob wir aus dieser Erfahrung Erkenntnisse für die deutsch-deutsche Entwicklung gewinnen können. Die Schwierigkeit meines Unterfangens besteht darin, einerseits so allgemein zu werden, daß wir quasi unmittelbar aus der Geschichte lernen; andererseits aber so spezifisch zu bleiben, daß sich unzulässige Übertragungen von selbst verbieten.

\section{*Wir sind doch kein besiegtes Land* (Die Zeit, Nr. 48 v. 23.11.90)}

Die garibaldinischen Freischärler, die 1860 Süditalien von den Bourbonen befreiten, hatten sich Illusionen über die Stimmung der Bevölkerung und über die eigene Stărke gemacht. Wenige Monate nach ihrem Siegeszug waren sie keine politische Kraft mehr, sondern nur noch moralische Aushängeschilder. Positiv gewendet: Garibaldi hatte zwar in der aktuellen Politik nichts mehr zu melden, blieb aber eine identitătsstiftende Leuchtschrift. Der Süden wurde de facto durch Piemont annektiert, das seine Gesetze und sein Verwaltungssystem auf den neuen Landesteil übertrug. In einer ersten Phase wurden die Einführung des Freihandels und das Abschütteln feudaler Fesseln - insbesondere die Privatisierung des Mischund Gemeinbesitzes an Grund und Boden - als Maßnahmen angesehen, die von selbst zu mittelständischem Fortschritt und Wohlstand führen würden. Aber den Preis der Privatisierung zahlten die armen Bauern, die nun das vergangene Regime verklărten und massenhaft zu reaktionären »Sozialrebellen« (Hobsbawm 1962) wurden. Daß diese nicht nur damals verklärt wurden, sondern auch heute, selbstverständlich unter ganz anderen historischen Verhältmissen, die Gefahr einer „Verklärung« der vergangenen Verhältnisse besteht, hat Günter Kunert angemahnt (in: Frankfurter Allgemeine Zeitung, 29.12.1990). Aus Gründen der politischen Stabilität und des Schutzes des Privateigentums unterstützten der Norden und seine neue Verwaltung in den Anschlußgebieten die alten, bisher bekämpften Machtstrukturen des Großgrundbesitzes. So wechselten lediglich Name und Couleur der Machthaber oder, wie Tommaso Di Lampedusa formulierte: »Alles mußte sich ăndern, damit alles beim alten bleiben konnte «. Der durch die Landverteilung entstandene neue agrarische Mittelstand blieb schwach und unter der politischen Vormundschaft der Latifundien.

\section{Privateigentum, Rechtssicherheit, Wirtschaftskriminalität}

Ein großer Teil der neuen Eigentumstitel an Grund und Boden blieb juristisch um= stritten. Da die neuen Gesetze und Vorschriften nur selektiv im Interesse der Begüterten gehandhabt wurden, erhielt die Rechtsunsicherheit einen Dauerstatus. Begüterte setzten ihre Ansprüche durch Korruption, soziale Aufsteiger oder durch bewaffnete Selbsthilfe durch. Die hilflosen Massen reagierten mit Rebellionen und mit gelegentlicher Brandschatzung der Katasterämter. Individueller Landhunger und Erinnerungen an den alten Gemeinbesitz wurden zu fixen Ideen des kol- 
lektiven Bewußtseins (Kammerer 1979). Wo kleine und mittlere Eigentumstitel durchgesetzt werden konnten, verwandelte sich der Süden in eine fruchtbare Gartenlandschaft. Der Großgrundbesitz blieb von uneingelösten sozialen Anspriichen belastet (Cinanni 1977) und wirtschaftlich unproduktiv.

Die chaotische und fragmentarische Auflösung der feudalen Eigentumsstrukturen wurde zum Nährboden und zum Betätigungsfeld einer neuen und lukrativen Wirtschaftskriminalität, der Mafia (Blok). Deren oft beschworenen uralten Traditionen sind nur ein Steinbruch, aus dem sich eine neue Aufsteigerschicht bediente. Die Mafia ist keineswegs ein feudales Überbleibsel, das unter voll entwickelten bürgerlich-kapitalistischen Verhältnissen verschwinden würde ${ }^{2}$, sondem eine hybride Mischung bürgerlich-kapitalistischer und feudaler Elemente, die im Treibhaus des italienischen Welfare erst richtig gedeiht. Die Mafia ist ein erfolgreiches »sowohl, als auch «, sie braucht den Markt und ist Marktersatz.

\section{Die Arbeitsteilung Nord-Süd}

Vom Freihandel und erweiterten inneren Markt profitierte zunächst die intensive kleine und mittelständische Landwirtschaft (Wein und Agrumen). Die Einführung von Schutzzöllen im Interesse der werdenden Industrie und des latifundistischen Getreideanbaus vernichtete die Exportchancen der intensiven und begünstigte die extensive Landwirtschaft des Großgrundbesitzes. Neapel war zum Zeitpunkt der Einigung ein ebenso entwickelter Industriestandort wie Genua. In Neapel war der erste Mittelmeerdampfer vom Stapel gelaufen (1818) und es wurde dort die erste italienische Eisenbahn in Betrieb genommen (1839). Aber der plötzliche Übergang zum Freihandel nach der Einigung hatte negative Auswirkungen. Es wurde im Keim erstickt, »was später nur wieder mit tausendfacher Mühe ins Leben gerufen werden konnte (Vöchting 1951, S. 218). Der Übergang zum Protektionismus 1887 kam dann zu spät und praktisch nur noch dem Norden zugute. So wurde in den ersten Jahrzehnten nach der Einigung politisch das Bündnis zwischen süditalienischem Großgrundbesitz und norditalienischer Industrie geschmiedet und damit in der Ökonomie eine Arbeitsteilung zwischen Landwirtschaft und Industrie eingeleitet und festgeschrieben, die dem Süden eine subalterne Rolle zuwies. Die ersten Sondergesetze zugunsten einzelner Regionen des Südens und zugunsten Neapels wurden folglich bereits 1904 erlassen.

\section{Die gespaltene Konjunktur}

Nach dem zweiten Weltkrieg deklarierte man unter dem Einfluß der damaligen

2 Die italienische Linke der $50 \mathrm{er}$ und 60er Jahre sprach von »feudalen Residuen«, die durch kapitalistische Methoden ersetzt werden könnten. Enzensberger (1978) folgte dieser Vorstellung in seinem spannenden Aufsatz über die Camorra aus dem Jahre 1960, ebenso der Verfasser (1973). In seinem Italien gewidmeten Kapitel in »Ach Europa « beschreibt Enzensberger großartig die Rolle feudaler Überreste als Lebenselixier der italienischen Entwicklung. 
amerikanischen entwicklungstheoretischen und -politischen Vorstellungen den Süden Italiens zum Entwicklungsland. In etwa 10 Jahren, so dachte man, kơnnte der Süden den Norden einholen, falls man die Fehler der Vergangenheit vermeiden würde: Bisher hatte der Norden aus dem Süden Kapital für seine eigene Entwicklung abgezogen, jetzt müßte das Umgekehrte passieren; bisher war die Arbeitsteilung Süd-Nord nach dem Stadt-Land-Modell festgeschrieben worden, jetzt müsse nach einer Phase der Landreform und der Infrastrukturinvestitionen die Industrialisierung des Südens erfolgen. Eine eigene Behơrde, die »Cassa per il Mezzogiorno«, sollte die Umverteilung der öffentlichen Mittel vornehmen, die Infrastruktur verbessern und Investitionszuschüsse an die private Industrie gewähren. Zu diesem Instrumentarium gehörten außerdem zinsverbilligte Kredite und fiskalische Maßnahmen. Doch schon nach wenigen Jahren wurde klar, daß der Süden den Norden nicht einholen, sondern bestenfalls gleiche Wachstumsraten wie der Norden erzielen konnte. Das ist bis heute so geblieben. Zwischen 1950 und 1986 stieg die süditalienische Wertschöpfung pro Kopf der Bevölkerung von $55 \%$ auf $60 \%$ derjenigen Norditaliens an, um bis 1989 wieder auf $56,4 \%$ abzusinken (SVIMEZ 1988; 1990). Im Verlauf einer Hochkonjunktur im Norden vergröBert sich jedes Mal der Abstand. Sichtbar wurde dies bereits beim großen Wirtschaftswunder in den Jahren von 1958 bis 1963. Zu diesem »Wunder«, und damit auch zur Vergrößerung des Abstands, hat der Süden entscheidend beigetragen. Die öffentlichen und privaten Ausgaben im Süden verwandelten sich zum großen Teil in Nachfrage nach im Norden hergestellten Gütern; die vom Süden in den Norden wandernden billigen Arbeitskräfte trugen zur internationalen Konkurrenzfähigkeit der norditalienischen Industrie bei. Selbst während der Hochphase des »Wirtschaftswunders (bis 1963) gab es keinen Anstieg der Reallöhne. Trotzdem wurde von liberaler Seite (Lutz 1962) die mangelnde Elastizität der Löhne nach unten für die fehlenden Investitionen im Süden verantwortlich gemacht.

Das Wirtschaftswunder Italiens hatte schon Mitte der 60er Jahre zu drei fundamentalen Ungleichgewichten geführt: (a) zur Orientierung der ärmeren Gebiete am Konsummodell der reichen Länder und damit auch zur Vernachlässigung kollektiver Bedürfnisse (Gesundheit, Verkehr, Ausbildung) zugunsten der Befriedigung gehobener privater Konsumwünsche; (b) zur Erweiterung des dualen »gap« zwischen der traditionellen arbeitsintensiven Industrie und dem modernen kapitalintensiven Sektor; (c) zur Vertiefung des Unterschiedes zwischen Nord und Süd. Nur eine demokratische Planung der Investitonstätigkeit könne den spontanen Marktkräften gegensteuern und diese Probleme lösen, meinte eine Mehrheit quer durch die Parteien - von den Kommunisten bis zu den Christdemokraten (vgl. die Beiträge zu Graziani 1978). Das Südproblem - so lautete ein neuer Konsens betreffe nicht nur den Süden; ein »einziger Mechanismus« (»il meccanismo unico《) erzeuge auf der einen Seite Wohlstand, auf der anderen aber Unterentwicklung (Libertini 1968). Eine Lösung der Südfrage erwartete man nicht mehr vom bloßen Transfer von Akkumulationsenergien, sondern man stellte das Akku- 
mulationsmodell selbst in Frage. Treibende Kraft einer prakrischen Kritik war die Arbeiterbewegung.

\section{Die hybride Modernisierung}

Bereits im Jahre 1957 wurde das Imperium der Untemehmen mit Staatsbeteiligungen (IRI, ENI, EFIM etc.) verpflichtet, 60\% ihrer Neuinvestitionen in SüditaLien zu tătigen. Das Ergebnis dieses im wesentlichen nie eingehaltenen Gesetzes und des Übergangs von der Konzeption einer feinmaschigen, arbeitsintensiven Investitions-förderung zu einer kapitalintensiven Schwerpunktbildung sind die sogenannten »Kathedralen in der Wüste« der Petrochemie und der Stahlindustrie. Die neue Arbeiterklasse dieser Industrieenklaven erkämpfte zusammen mit den Arbeitem des Nordens die Beseitigung der sogenannten "gabbie salariali«, der territorialen Lohnabstufungen. Gleiche Löhne im Norden wie im Süden sollten die Abwanderung von Arbeitskräften eindämmen und die Industrie zwingen, beabsichtigte Neuinvestitionen in den Süden zu verlegen, was dann auch in der Periode zwischen 1969 und 1973 tatsächlich passierte. Ausschlaggebender Faktor: die Industrie wollte den Arbeitskonflikten im Norden ausweichen. Der Anteil des Südens an den Industrieinvestitionen Italiens stieg in diesen 4 Jahren von 28,1\% auf 33,5\%. Die sogenannte Ölkrise und die anschließende Stahlkrise haben dann Mitte der 70er Jahre diese Industrialisierung abrupt gestoppt. In den 80er Jahren fiel der Anteil des Sủdens an den industriellen Investitionen des Landes unter $25 \%$. Wie zum Trost entdeckte man die postmoderne Blüte der Schattenwirtschaft.

Wer sich Ende der 70er Jahre mit dem Mezzogiomo beschäftigte, stand vor einem konzeptionellen Trümmerhaufen. Keine der hochgespannten Erwartungen war eingetreten. Weder die geradlinigen Modernisierungs- und Rationalisierungsvorstellungen der technokratischen Denker in Entwicklungsstadien, noch die politökonomischen Vorstellungen von dialektischen Umwälzungen gaben ein brauchbares Bild der Wirklichkeit. Ein Paradigmenwechsel war fällig. Der Süden erscheint dem heutigen Beobachter als hybride Mischung, als ein Chaos: enorm resistent gegen Neuerungen und doch deren leichte Beute; rebellisch, aber christdemokratisch; anomisch und durchzogen von sozialen Bindungen; eine Überflußgesellschaft, der es am Nötigsten fehlt, nämlich an Arbeit; erdbebengebeutelt und postindustriell; ein Eldorado staatlich geförderter Schattenwirtschaft mit der Mafia als größtem Konzem (Arlacchi 1986), der umsatzmäßig inzwischen selbst FIAT überrundet hat. Anständige Politökonomen wenden sich von diesem Bild mit Grausen und überlassen das zerklüftete Forschungsfeld den Anthropologen und soziologischen Abenteurem. Der Süden hat anscheinend im Übergang vom Feudalismus zum Kapitalismus einen eigenen, dritten Weg entdeckt. Oder vorsichtiger: einen eigenen Wirtschaftsstil, der nun seinerseits nordwărts dringt. »Die Palmengrenze verschiebt sich nach Norden«, schrieb Sciascia. 


\section{Interpretationspfade ins Chaos}

Wir sind weit von deutschen Verhältnissen entfernt. Und doch habe ich den Eindruck, $d a B$ einige der ins süditalienische Chaos vorangetriebenen Interpretationspfade für die Klärung auch unserer Verhältnisse von unmittelbarer Bedeutung sein können.

Der regelmäßige und riesige Einkommenstransfer hat die suiditalienische Klassenstruktur verändert. Den Geldkanälen entlang, die von Rom ausgehend bis ins kleinste Dorf führen, hat sich nicht nur eine Bürokratie, sondern auch eine ganze Schicht von Vermittlern und Agenten gebildet (Gribaudi 1980). Diese vermarkten ihr Wissen und ihre Beziehungen und spielen die Makler im Tauschgeschäft, in dem es um Partizipation an politischer Macht und Partizipation an öffentlichen Mitteln geht. An diese Mittel heranzukommen ist für fast alle Unternehmen erfolgsentscheidend und für weite Teile der Bevölkerung eine Existenzfrage. Ähnliches gilt für den Arbeitsmarkt. Nur im Netzwerk von weiten Familien- und Klientelbeziehungen sind Arbeitsplätze zu finden (Bechtle-Künemund 1988). Die begehrten Arbeitsplătze und Tätigkeitsfelder liegen nicht in der Produktion, sondern im formellen und informellen Apparat zur Kontrolle und Verteilung offentlicher Mittel. Dieser Apparat, zu dem auch ein guter Teil der organisierten Kriminalität gehört, spielt die zentrale Rolle in der süditalienischen Wirtschaft und Gesellschaft. Er hegemonisiert die gesamte Produktionsstruktur. Die Allokation von Geld, Kredit und Arbeit erfolgt zwar über Märkte, deren Funktionsweise und System haben jedoch wenig mit unseren Vorstellungen von freier Marktwirtschaft zu tun. Augusto Graziani, einer der bedeutendsten Ökonomen Italiens, beschrieb das kürzlich so: „Wir bezeichnen diese Strukturen hartnäckig als Marktwirtschaft, aber in Wirklichkeit handelt es sich um das modernisierte Spiegelbild einer feudalen Wirtschaft - mit dem Unterschied, daß die Rolle des Bodens nun durch die öffentlichen Ausgaben eingenommen wird « (Graziani in »ll Manifesto«, 14.4.1990). Wenn Graziani recht hat bedeutet das, daß das Ergebnis der Einführung einer freien Marktwirtschaft nicht unbedingt eine freie Marktwirtschaft sein muB.

\section{Fazit}

Ich nehme an, daß diese Gedankengänge eine größere Rolle für Osteuropa als für Ostdeutschland spielen könnten. Aber auch nur auf Deutschland angewandt sind sie geeignet, den gegenwärtigen breiten Konsens fragwürdig zu machen, der sich in den folgenden Sătzen präsentiert: »Wir wollen das westliche Modell. Es gibt keinen 3. Weg. Keine Experimente« (Die Zeit v. 23.11.90). Das Beispiel Italien zeigt, daß es das »westliche Modell« tout court nicht gibt, daß die kapitalistische Wirklichkeit selbst in einem Land wesentlich differenzierter ist als gemeinhin angenommen wird. Die relativ homogene Bundesrepublik ist eher eine Ausnahme als die Regel und sie wird durch den Einigungsproze $B$ differenzierter und komplexer. Denn die Einführung der Marktwirtschaft im Beitrittsgebiet vollzieht sich ja 
nicht im luftleeren Raum, sondern auf dem Boden mehr oder weniger resistenter materieller und immaterieller Strukturen. Gerade der heute so mächtig wirkende Rückgriff auf die Geschichte vor der Gründung der DDR zeigt, welche Rolle die Vergangenheit für die Zukunft spielt. Zur wirksamen Vergangenheit gehort aber auch die Geschichte der DDR. Sie einfach verdrängen oder ausschalten zu wollen, führt emeut unter eine experimentelle Käseglocke. Unter der Käseglocke des real existierenden Sozialismus entwickelten sich hybride Mischungen, z.B. Refeudalisierungsprozesse unter sozialistischem Vorzeichen (so beispielsweise Jürgen Kuczynski). Was passiert unter der Käseglocke der freien Marktwirtschaft mit dem Experiment, das bereits in vollem Gang ist?

\section{Literatur}

Arlacchi, Pino (1986): Die Mafia und das internationale Geldsystem, in: PROKLA 63, 16. Jahrgang 1986, Nr. 2, S. 63-69

Bechtle-Künemund (1988): Rückkehr nach Monopoli. Spielregeln eines lokalen sïditalienischen Ar. beitsmarkes, Frankfurt und New York

Cinanni, Paolo (1977): Lotte per la terra e comunisti in Calabria 1943/1953, Milano

Enzensberger, Hans Magnus (1978): Politik und Verbrechen, Frankfurt

Enzensberger, Hans Magnus (1987): Ach Europa, Frankfurt

Gribaudi, Gabriella (1980): Mediatori. Antropologia del potere democristiano nel Mezzogiorno, Torino

Graziani, Augusto, Hrsg. (1978): L'economia italiana, 1945-1975, Bologna

Kammerer, Peter (1973): Gründerjahre und mehr über die Herren Peachum und Mackeath, Nachwort zu Chotjewitz, Peter: Malavita, Köln

Kammerer, Peter (1979): Bauernkämpfe und Landreform 1943-1953 in Süditalien, in: Poppinga, Onno (Hrsg): Produktion und Lebensverhältonisse auf dem Land, Leviathan Sonderhef 2, Opladen

Libertini, Lucio (1968): Integrazione capilalitica e soltosviluppo, Bari

Lutz, Vera (1962): Italy - A Study in Economic Development, London, New York, Toronto

SVIMEZ (1988, 1990): Un programma per il Mezzogiomo, Rapporto 1988. Bologna, Rapporto 1990. Roma

Vöchting, Friedrich (1951): Die italienische Südfrage, Berlin 